\title{
ENTEROPARASITOSES EM CRIANÇAS DE UMA CRECHE NA CIDADE DE ASSIS/SP - ANTES E DEPOIS DE CAMPANHAS EDUCATIVAS
}

\author{
ENTEROPARASITOSIS IN CHILDREN OF A CRECHE IN THE CITY \\ OF ASSIS: BEFORE AND AFTER EDUCATIONAL CAMPAIGNS
}

\section{Karin Maria Ludwig' e Adriane de Oliveira Campos Conte ${ }^{2}$}

\section{RESUMO}

As parasitoses intestinais estão relacionadas às condições sanitárias e as crianças compõem um grupo com elevada frequência de enteroparasitoses. $O$ objetivo deste trabalho foi realizar um levantamento da prevalência de enteroparasitos em alunos de uma creche da cidade de Assis-SP, junto a campanhas educativas sobre métodos de profilaxia. Métodos - A análise das fezes foi realizada pelos métodos de Hoffmann, Pons e Janer e Faust. A primeira análise foi realizada em 136 crianças da creche. Na segunda etapa do trabalho foram realizadas campanhas de educação sanitária e profilática com os pais e com as crianças da creche e posteriormente nova coleta e análise das amostras de fezes. Resultados - 0 índice de parasitoses geral foi de $27,9 \%$. Verificou-se uma maior incidência na faixa etária de 2 a 6 anos, destacando- se a idade de 5 a 6 anos. Foram realizadas campanhas de educação sanitária e profilática junto aos pais, funcionários e crianças da creche. Na segunda coleta houve uma diminuição na incidência, com 11,8\%.

Conclusões - Concluiu-se que as campanhas educativas são de grande importância, mas precisam ser integradas a um processo contínuo de educação a fim de controlar e combater as enteroparasitoses.

Descritores: enteroparasitoses, creche, profilaxia, campanhas educativas, Assis.

\section{ABSTRACT}

Intestinal parasitosis are related to sanitary conditions, and children make up a group with high frequency of enteroparasitosis. The aim of this study was to survey the prevalence of enteroparasites in children of a creche in the city of Assis-SP, along with educational campaigns on prophylactic methods. Methods - Stool analyses were carried out by using Hoffman, Pons and Janer, and Faust methods. The first analysis was performed on 136 children of the creche. In the second stage of the work, sanitary and prophylactic educational campaigns were conducted among the parents and children of the creche and, after that, there was a second stool collection and analysis. Results - The overall level of parasitosis was $27.9 \%$. There was a higher incidence in the age group of 2-6 year-olds, mainly in the age group of 5 to 6 year-olds. Sanitary and prophylactic educational campaigns were carried out among the parents, staff and children of the creche. In the second collection there was a decrease in incidence of $11.8 \%$. Conclusions - It was concluded that educational campaigns are of great importance, but they need to be integrated into a continuous awareness process in order to control and fight enteroparasitosis.
${ }^{1}$ Formada em Ciências Biológicas pela Universidade Estadual Paulista (UNESP), Assis, SP, Brasil

${ }^{2}$ Formada em Farmácia e Bioquímica pela Universidade Paulista (UNIP), Assis, SP, Brasil

Descriptors: enteroparasitosis, creche, prophylaxis, educational campaigns, Assis. 


\section{Introdução}

Aproximadamente um terço da população das cidades dos países em desenvolvimento vive em condições ambientais propícias à disseminação das infecções parasitárias. Embora apresentem baixas taxas de mortalidade, as parasitoses intestinais ainda continuam representando um problema significativo de saúde pública, haja vista o grande número de indivíduos afetados e as várias alterações orgânicas que podem provocar, inclusive sobre o estado nutricional ${ }^{1-2}$.

As parasitoses intestinais estão intimamente relacionadas às condições sanitárias e representam um importante problema de saúde pública nos países em desenvolvimento ${ }^{3-5}$. Estas infecções podem estar relacionadas ainda a educação sanitária, noções de higiene e aspectos culturais mais deficitários que apresentam relevância no controle dessas doenças ${ }^{6}$.

Estudos realizados apontam o Brasil como um dos países com grave problema de saúde pública devido à carência de saneamento básico somado com a falta de conhecimento da população com relação à higiene pessoal. Devido a isso, o índice dessas parasitoses alcança nível significativo em crianças em idade escolar, sendo a maioria dos casos localizados na periferia das cidades ${ }^{4,7}$.

Os danos que os parasitos podem causar a seus portadores incluem, entre outros agravos, a obstrução intestinal (Ascaris lumbricoides), a desnutrição (Ascaris lumbricoides e Trichuris trichiura), a anemia por deficiência de ferro (ancilostomídeos) e quadros de diarreia e de má absorção (Entamoeba histolytica e Giardia lamblia), sendo que as manifestações clínicas são usualmente proporcionais à carga parasitária albergada pelo indivíduo ${ }^{5,8-9}$.

As crianças são as mais acometidas, podendo a maior prevalência de parasitas intestinais levar a déficit nutricional e do desenvolvimento físico e intelectual, além de problemas gastrintestinais, ansiedade, nervosismo e consequente atraso no desenvolvimento escolar ${ }^{3,10-12}$.

A transmissão desses parasitos é geralmente orofecal, ou seja, a ingestão de ovos embrionados ou cistos, através de alimentos, contato pelas mãos, água e até poeira contaminada ${ }^{4,13}$

Devido a maior participação feminina no mercado de trabalho, as creches passaram a ser o primeiro ambiente externo ao doméstico que a criança frequenta, tornando-se potenciais ambientes de contaminação 3,5, 13-14.

As creches são lugares propícios para essas infecções, partindo do princípio de que são lugares que possuem grande quantidade de crianças em ambientes fechados e com alto contato inter-humano ${ }^{5,15-17}$.

O parasitismo intestinal em crianças que frequentam creches tem sido estudado por diferentes autores, destacando a necessidade de campanhas educativas de prevenção tais como: higiene, educação sanitária, saneamento básico, alimentação e terapêutica, bem como desapertá-la para o controle das doenças parasitárias, incluindo-as nos programas dos educadores ${ }^{15-16,18}$. Assim, tem que se aproveitar deste espaço, pois a creche, como uma instituição social, tem como objetivo educar e formar a criança que, na maior parte do tempo, está sob cuidados dos educadores, ou monitores ${ }^{14}$.

Assim, esta pesquisa teve por objetivo verificar a prevalência de enteroparasitoses na creche e a execução de campanhas educativas junto aos pais e alunos da escola, a fim de conscientizá-los, quanto os mecanismos de transmissão, profilaxia e tratamento de cada uma das enteroparasitoses conhecidas e verificar o efeito deste tipo de intervenção nesta comunidade.

\section{Metodologia}

A presente pesquisa foi desenvolvida em uma creche localizada no bairro Vila Operária da cidade de Assis, estado de São Paulo, nos anos de 2006 e 2007. Inicialmente foram realizadas reuniões com os professores, monitores, direção e funcionários da escola e posteriormente com os pais de alunos a fim de apresentar informações do trabalho, como os objetivos, etapas e principalmente sensibilização da comunidade escolar para participação.

Após a aprovação do Comitê de Ética em Pesquisa da Universidade Paulista (protocolo 216/06) os pais e/ou responsáveis receberam um termo de consentimento livre e esclarecido, autorizando a participação das crianças no estudo.

Cada criança recebeu um coletor identificado e um explicativo para procedimento da coleta das amostras. As fezes foram colhidas em dias pré-determinados, e os exames de fezes realizados utilizando dois métodos para cada amostra: Hoffmann et al (1934) $)^{19}$ conhecido como método de sedimentação espontânea e o método de centrifugoflutuação de Faust et al (1938) ${ }^{20}$, sendo utilizado o Laboratório de Análises Clínicas da Universidade Paulista. 
Posteriormente foram realizadas campanhas de educação sanitária e profilática com os pais e com as crianças da creche através da apresentação de cartazes explicativos, onde se esclareceu as formas de transmissão, profilaxia dos principais parasitos encontrados, ressaltando sempre a importância de alguns cuidados básicos de higiene domiciliar e pessoal a fim de conscientizá-los. Posteriormente, com as crianças utilizou-se um vídeo de animação sobre parasitoses intestinais: "Campanha de Combate a Verminose" produzido para empresa Janssen-Cilag (200-?) ${ }^{21}$ a fim de estimulá-los. Após a apresentação do filme, foram feitas algumas atividades sobre este para as crianças para fixação, onde houve uma participação muito importante da parte destas.

Em seguida realizou-se a $2^{\mathrm{a}}$ coleta de fezes das crianças que entregaram a $1^{\mathrm{a}}$ amostra, para uma comparação de resultados.

Após análise das fezes foram encaminhados aos pais e/ou responsáveis os resultados do exame parasitológico para que os mesmos procurassem o tratamento adequado nos casos positivos, contando com a colaboração das Unidades Básicas de Saúde Vila Operária e Vila Maria Izabel.

\section{Resultados e Discussão}

Foram analisadas 136 amostras e destas 38 estavam positivas para um ou mais parasitas intestinais ou comensais, e 98 negativos perfazendo $27,9 \%$ e $72,1 \%$ respectivamente (Figura 1 ).

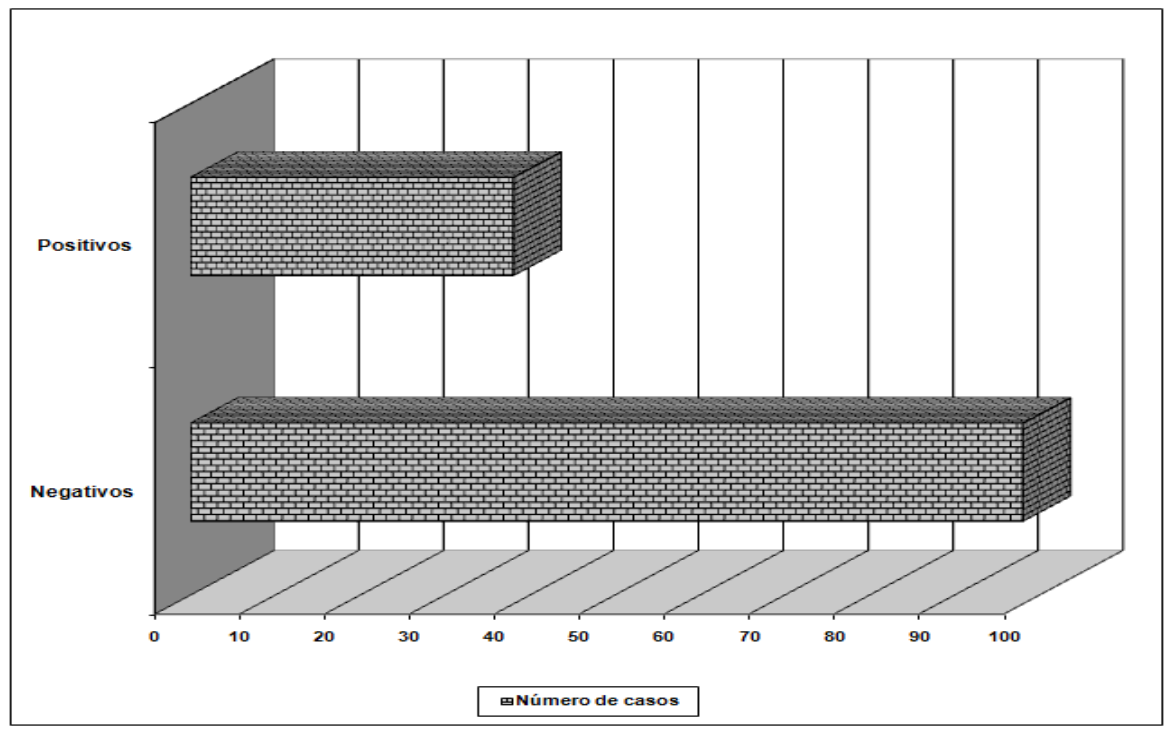

FIGURA 1 - Frequência de casos positivos e casos negativos na amostra de crianças da creche na primeira coleta, antes das atividades de educação sanitária.

Tal incidência assemelha-se aos resultados sobre pré-escolares de Duas Vassouras - RJ onde a positividade foi de $26,19 \%{ }^{2}$. Assemelha-se, também, aos resultados de Vespasiano - MG no qual os autores obtiveram incidência de $22,7 \%{ }^{12}$ e um estudo em Belo Horizonte - MG, que obteve uma incidência de $24,6 \%$ de parasitados ${ }^{22}$, enquanto no estudo realizado em centros de educação infantil de São Mateus, no Estado Santo do Espírito, a incidência geral foi de $36,2 \%{ }^{17}$, ligeiramente superior ao resultado obtido no presente estudo. Os autores ${ }^{17}$ explicam esses resultados pelo fato das creches e escolas serem um lugar onde há aglomerado de pessoas, sendo que assim, a transmissão acaba sendo facilitada através do contato de pessoa a pessoa.

A distribuição dos casos positivos foi analisada segundo duas categorias: monoparasitismo e biparasitismo. Houve predominância de monoparasitismo (33 casos) em relação à ocorrência de biparasitismo (5 casos), cujas espécies estão relacionadas na tabela 1. Dentre as crianças as crianças que apresentaram biparasitismo a associação mais frequente foi entre Giardia lamblia e Entamoeba coli. O protozoário Giardia lamblia aparece em 4 associações enquanto que o comensal Entamoeba coli aparece em três, seguido pelos helmintos Ascaris lumbricoides em 2 casos e Enterobius vermiculares em 1 caso. Estes resultados são similares aos obtidos por Tashima e Simões (2005) ${ }^{8} \mathrm{em}$ estudo realizado de prevalência e correlação com a idade e com os sintomas apresentados de uma população infantil de Presidente Prudente-SP. 
De acordo com a tabela 1 e Figura 2, dos helmintos, os mais frequentes foram: Hymenolepis nana (21\%); Ascaris lumbricoides (10,5\%); Enterobius vermicularis (10,5\%); Ancylostomidae (2,63\%) para os protozoários parasitas os mais frequentes foram: Giardia lamblia $(13,1 \%)$ e Entamoeba histolytica $(7,8 \%)$ e 0 protozoário comensal Entamoeba coli (21\%).

Entre os comensais o único encontrado foi Entamoeba coli com prevalência de $21 \%$. Valor superior ao estudo realizado por Ludwig et al (2012) ${ }^{4}$ onde observou-se um resultado de 4,8\% em um levantamento realizado num bairro da cidade de Cândido Mota/SP. O encontro deste comensal é um indicador de baixas condições de higiene e contaminação fecal. Também indica risco de infecção por outros agentes patogênicos que possuem o mesmo tipo de disseminação 22-24. É importante ressaltar que sendo ele um comensal, não provoca danos ao hospedeiro e consequentemente não apresenta nenhum sintoma ${ }^{8}$.

Foi observada uma incidência baixa para Entamoeba hystolitica (3 casos) semelhante ao estudo realizado por Tashima e Simões $(2005)^{8}$, onde há apenas 1 caso deste parasito. Segundo informações, à prevalência de amebíase intestinal por faixa etária é controversa, em algumas regiões se mostra elevada enquanto em outras a incidência mais baixa.

TABELA 1 - Distribuição dos helmintos, protozoários parasitos intestinais e protozoários comensais encontrados de acordo com a faixa etária, antes dos trabalhos de educação sanitária.

\begin{tabular}{|c|c|c|c|c|c|c|c|c|}
\hline Faixa & \multicolumn{4}{|c|}{ Helmintos } & \multicolumn{2}{|c|}{ Protozoários } & Protozoário & Biparasitismo \\
\hline & $\mathrm{Al}$ & $\mathrm{Hn}$ & Ev & A & Eh & $\mathrm{Gl}$ & Ec & - \\
\hline $0-1$ & - & - & - & - & - & - & - & - \\
\hline $1-2$ & - & 1 & - & 1 & - & - & 1 & - \\
\hline $2-3$ & - & 3 & 1 & - & - & - & - & $\begin{array}{c}1 \text { (Gl e Ec); } 1 \text { (Gl } \\
\text { e Ev) }\end{array}$ \\
\hline 3-4 & - & - & 1 & - & 1 & 2 & 1 & 1 (Al e Ec) \\
\hline 4-5 & 3 & 2 & 1 & - & - & - & 1 & $\begin{array}{c}1 \text { (Gl e Ec); } 1 \text { (Gl } \\
\text { e Al) }\end{array}$ \\
\hline $5-6$ & 1 & 1 & - & - & 2 & 2 & 4 & - \\
\hline $6-7$ & - & - & - & - & - & - & 1 & - \\
\hline $7-8$ & - & 1 & 1 & - & - & 1 & - & - \\
\hline Total & 4 & 8 & 4 & 1 & 3 & 5 & 8 & 5 \\
\hline
\end{tabular}

Al. Ascaris lumbricoides; Hn. Hymenolepis nana; Ev. Enterobius vermicularis; A. Ancylostomidae; Eh. Entamoeba histolytica; Gl. -Giardia lamblia; Ec. Entamoeba coli 


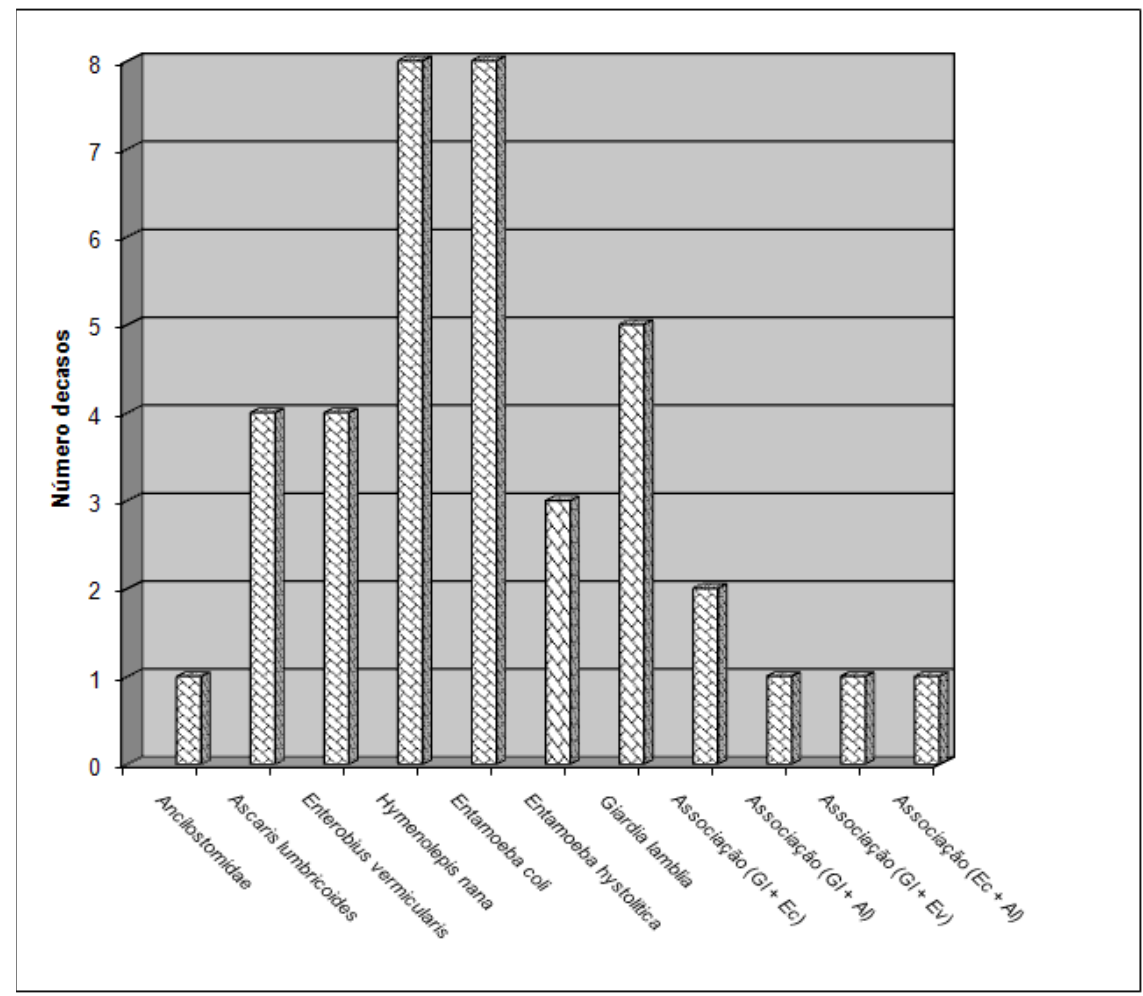

FIGURA 2. Distribuição geral de protozoários e helmintos encontrados nas amostras de exames de fezes na primeira coleta, antes das atividades de educação sanitária.

Observou-se menor incidência de Ascaris lumbricoides (10,5\%) em relação á Giardia lamblia (13,1\%). Tal incidência ocorre pelo baixo número de indivíduos contaminados ou por que os ovos de Ascaris lumbricoides requerem um período de maturação de pelo menos três semanas em solo úmido e sombreado antes de se tornarem infectantes. Em relação à Giardia lamblia, seus cistos já são infectantes no momento de sua eliminação pelas fezes, o que permite transmissão pessoa-pessoa dispensando a passagem pelo solo e tornando possível o contágio de crianças mesmo em ambientes saneados como creches e pré-escola ${ }^{24}$.

No caso da Enterobíase, observou-se 4 casos, incidência baixa semelhante ao estudo realizado por Bencke et al $(2006)^{25}$, que encontrou apenas 5 casos. 0 autor sugeriu que tal ocorrência pode estar relacionada com o fato de a técnica utilizada não ser apropriada para o seu diagnóstico. Porém, a constatação da presença de ovos de Enterobius vermicularis nos exames indica a elevada carga parasitária nestas crianças e a grande probabilidade de que muitas outras estejam contaminadas por este. Ao contrário, em outro estudo realizado, o helminto mais frequente é o Enterobius vermicularis que observou 19 casos, onde a maioria não apresenta nenhum sintoma, porque o indivíduo só nota que alberga o parasita quando sente um prurido anal, principalmente à noite ${ }^{8}$.

A baixa incidência de Ancylostomidae na amostra, apenas 1 (2,63\%) foi menor do que os casos de Ascaris lumbricoides $(10,5 \%)$ e Giardia lamblia $(13,1 \%)$ o que coincide com os resultados de Mamus et al $(2008)^{26}$, cuja porcentagem de Ancylostomidae é de 3,12\%. Isso ocorre principalmente em crianças de 0-5 anos, devido aos hábitos precários de higiene e/ou imunidade baixa ${ }^{26}$.

As crianças estão mais expostas à contaminação em função do desconhecimento dos princípios básicos de higiene e do intenso contato com o solo, levarem as mãos sujas à boca e, muitas vezes, sem que os pais e responsáveis percebam, alimentam-se sem lavar as mãos. Ainda é importante levar em consideração que o sistema imunológico das crianças está menos apto a reconhecer e combater estes agentes patogênicos ${ }^{4,27}$.

Após o levantamento de dados da $1^{\text {a }}$ coleta foram realizadas as campanhas de educação sanitária e profilática com as crianças da creche, esclarecendo as formas de transmissão, profilaxia dos principais parasitos encontrados, ressaltando sempre a importância de alguns cuidados básicos de higiene. De acordo com Gelatti et al (2013) ${ }^{16}$ a educação é comprovadamente uma medida profilática efetiva. Assim, escolheu-se as campanhas para verificar a sua eficácia na referida creche. 
No Brasil estão descritas várias experiências bem sucedidas de educação para prevenção de doenças parasitológicas, entre elas, um trabalho realizado em Maringá-PR, no qual se verificou que após o tratamento educativo, por meio de minicursos para as famílias atendidas, ocorreu redução na prevalência para parasitos intestinais de $42,5 \%$ para $12,6 \%{ }^{23}$.

Medidas simples como a integração de hábitos de higiene, lavagem das mãos e dos alimentos com água e sabão, têm sido eficazes no combate às infeç̧ões causadas por parasitos; além do uso de um sapato e cuidados com as unhas têm sido importante na prevenção de parasitoses humana. Para efetivação destas medidas se faz importante a educação da população, que se consegue com campanhas de educação sanitária e ambiental bem estruturadas $15-16,18,28$.

Foi observado que após a realização de campanhas de educação sanitária e profilática com os pais e crianças e o devido tratamento das crianças parasitadas, houve uma diminuição no índice de positividade destes parasitos. Após as campanhas educativas foi realizada a $2^{\text {a }}$ coleta, onde apenas 6 crianças apresentaram resultado positivo para um parasito intestinal ou comensal, e 45 negativos perfazendo 11,8 e 88,2\%, respectivamente (Figura 3).

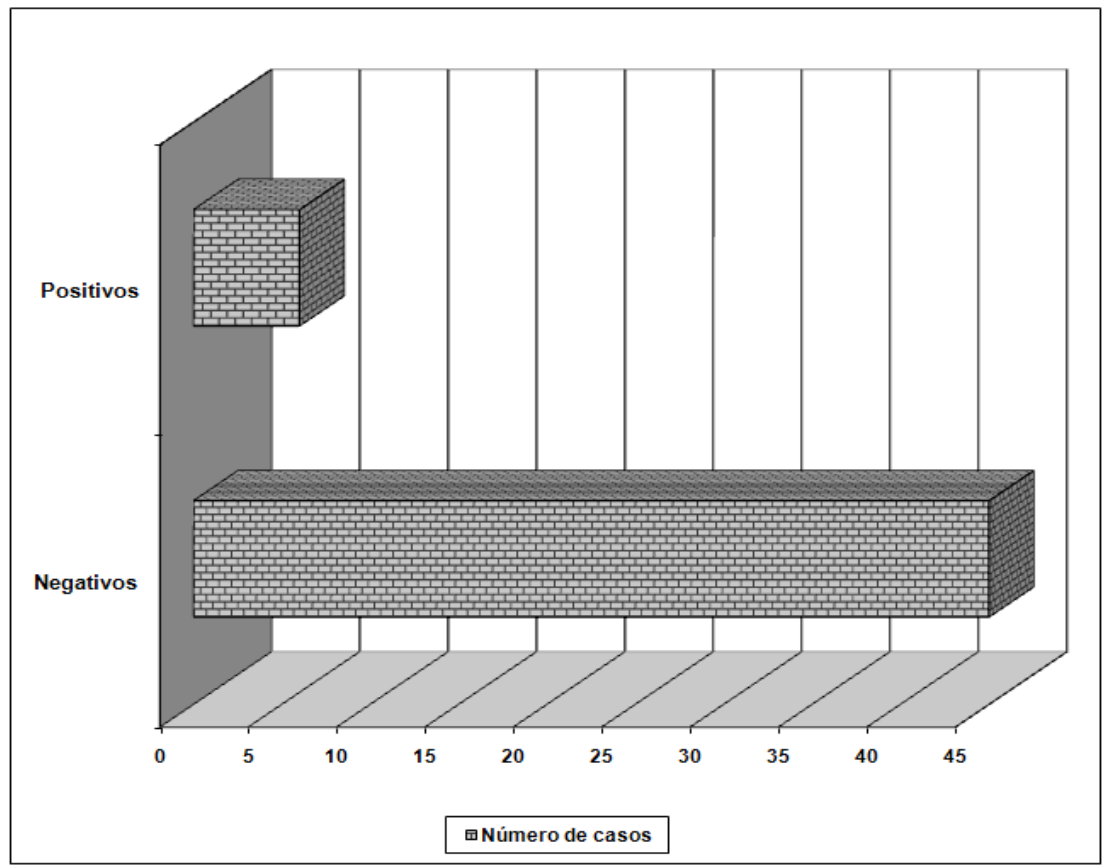

FIGURA 3 - Número de casos positivos e casos negativos da $2^{\text {a }}$ coleta das amostras das crianças da creche, após as campanhas educativas.

A maior incidência foi para o protozoário Giardia lamblia (4), sendo que esta incidência pode estar relacionada á condições higiênicas mais precárias e a instituições fechadas como creches (figura 4). Podendo a infecção provir do consumo de água poluída com dejetos humanos ou das mãos sujas de indivíduos infectados ao prestar serviços pessoais ${ }^{2}$.

O comensal Entamoeba coli e o protozoário Entamoeba histolytica tiveram incidência baixa, apenas 1 caso, e na $1^{\mathrm{a}}$ coleta a incidência de (8) e (3) respectivamente. $\mathrm{O}$ comensal E. coli indica baixas condições de higiene e contaminação fecal ${ }^{23-24}$. 


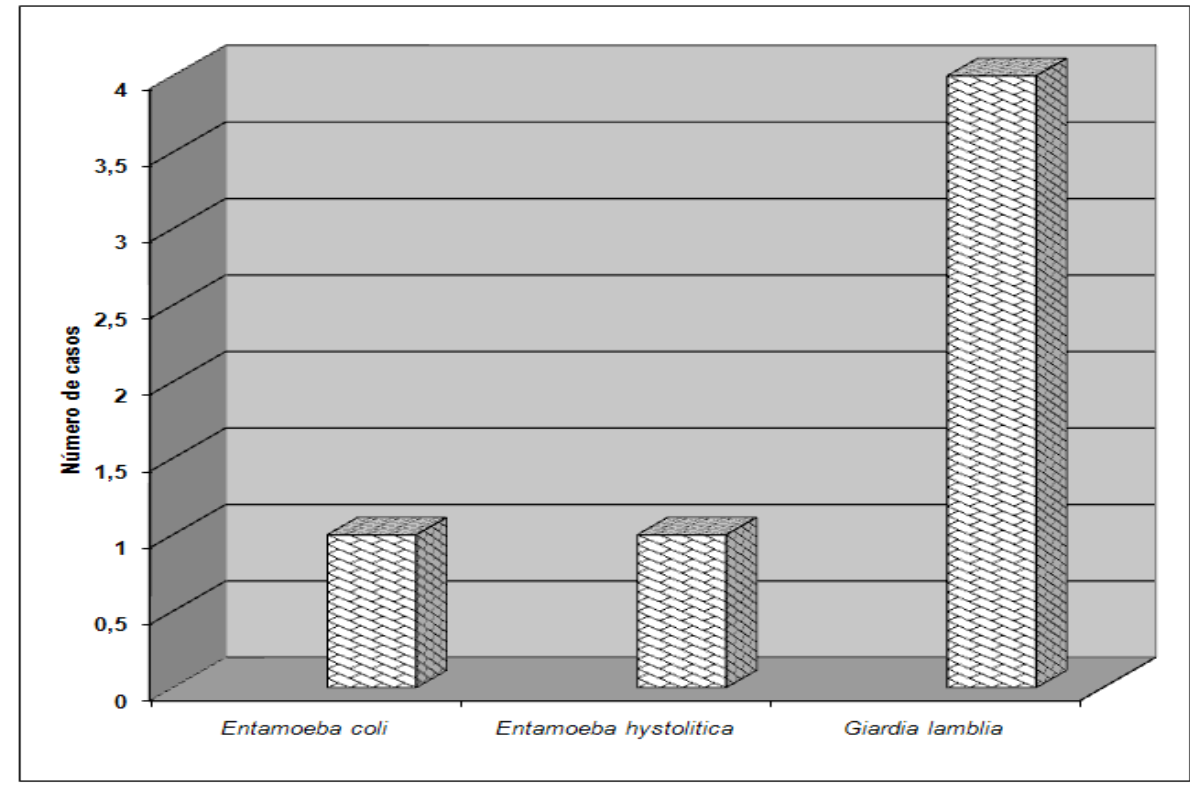

FIGURA 4. Distribuição geral de protozoários e helmintos encontrados nas amostras de exames de fezes na primeira coleta, após dos trabalhos de educação sanitária.

Relacionando-se as crianças de casos positivos na $2^{\mathrm{a}}$ coleta com a $1^{\mathrm{a}}$, e observou-se que dos 6 casos positivos na $2^{a}$ coleta apenas 1 crianças se encontrava parasitada no $1^{\circ}$ exame, mas por outro tipo de parasito. Havendo então outra infecção, discutiu-se a possibilidade de infecção por parte das crianças que estavam infectadas e não participaram da $1^{\text {a }}$ coleta, e a transmissão por parte dessas já que essas não foram devidamente tratadas.

Conforme citado após as campanhas educativas houve uma diminuição no índice de positividade, mas não sua total erradicação, fator que pode ser explicado por Kaliberda et al $(2008)^{29}$, onde diz que a erradicação desses parasitos requer melhorias das condições socioeconômicas, no saneamento básico e na educação sanitária. Isto nos mostra a importância de campanhas de educação sanitária, pois mesmo em população que reside em áreas altamente urbanizada e saneada, falta-lhes educação sanitária.

As práticas educacionais bem aplicadas levam as pessoas a adquirirem os conhecimentos para prevenção de parasitoses. $O$ valor da orientação pedagógica para a conscientização da população é imenso. Campanhas de orientação especialmente sobre a prevenção das parasitoses tornam-se essencial, evitando-se, assim, danos à saúde infantil decorrentes da falta de conhecimento sobre essas enfermidades por parte da família e da escola ${ }^{15,30}$.

\section{Considerações Finais}

Os exames foram desenvolvidos para diagnosticar as enteroparasitoses na creche juntamente com a execução de campanhas educativas e profiláticas. Assim, com a execução deste estudo foi possível observar que a incidência de enteroparasitoses entre as crianças da creche na $1^{\text {a }}$ coleta foi de $27,9 \%$, onde os enteroparasitos mais encontrados foram: Hymenolepis nana, Ascaris lumbricoides, Enterobius vermicularis, Ancylostomatidae, Giardia lamblia e Entamoeba histolytica. A incidência de enteroparasitoses entre as crianças da creche na $2^{a}$ coleta foi de $11,8 \%$, considerando-se que houve um bom aproveitamento por parte dos pais e das crianças em relação a campanhas de educação sanitária e profilática e o devido tratamento das crianças infectadas na $1^{\text {a }}$ coleta. Não havendo provavelmente, reinfecção por parte dessas.

Assim, os resultados da pesquisa fornecem dados sobre a importância das campanhas educativas podendo servir como um instrumento para auxiliar na elaboração de um plano de conscientização da população quanto aos bons hábitos de higiene em todas as suas vertentes. Importante dar ênfase as crianças que são as mais acometidas pelas enteroparasitoses e ainda por serem veiculadoras de informação e as transmitirem aos indivíduos que vivem ao seu redor. 


\section{Agradecimentos}

Este trabalho foi financiado pelo Conselho Nacional de Desenvolvimento Científico e Tecnológico - CNPq. Agradecemos a creche e aos participantes por ter possibilitado a realização deste trabalho

\section{Referências}

1. Oliveira CB, Silva AS, Monteiro SG. Ocorrência de parasitas em solos de praças infantis nas creches municipais de Santa Maria - RS, Brasil. Rev FZVA. 2007; 14(1): 174-179.

2. Pinheiro RO, Breguêz JMM, Baptista SC, Teixeira JL, Silva GMS. Ocorrência de parasitas intestinais entre crianças do pré-escolar de duas escolas em Vassouras, RJ. Rev Bras Farm. 2007; 88 (2): 98-99.

3. GURGEL RQ, CARDOSO GS, SILVA AM, SANTOS LN, OLIVEIRA RCV. Creche: ambiente expositor ou protetor nas infecções por parasitas intestinais em Aracaju, SE. Rev Soc Bras Med Trop. 2005, 38(3): 267-269.

4. Ludwig KM, Ribeiro ALT, Conte AOC, Decleva DV, Ribeiro JTD. Ocorrência de Enteroparasitoses na População de um Bairro da Cidade de Cândido Mota - Estado de São Paulo. J Health Sci Inst. 2012; 30(3): 271-276.

5. Santos J, Duarte ARM, Gadotti G, Lima LM. Parasitoses intestinais em crianças de creche comunitária em Florianópolis, SC, Brasil. Rev Patol Trop. 2014, 43(3): 332-340.

6. Visser S, Giatti LL, Carvalho RAC, Guerreiro JCH. Estudo da associação entre fatores socioambientais e prevalência de parasitose intestinal em área periférica da cidade de Manaus (AM, Brasil). Rev. Ciênc. saúde coletiva. 2011; 16(8): 3481-3492.

7. Silva JC, Furtado LFV. Ferro TC, Bezerra KC, Borges EP, Melo ACF. Parasitismo por Ascaris lumbricoides e seus aspectos epidemiológicos em crianças do Estado do Maranhão. Rev Soc Bras Med Trop. 2011; 44(1):100-102.

8. Tashima NT, Simões MJS. Parasitas intestinais; prevalência e correlação com a idade e com os sintomas apresentados de uma população infantil de Presidente Prudente - SP. Rev Bras Anal Clin. 2005; 37(1): 35-39.

9. Fonseca EOL, Teixeira MG, Barreto ML, Carmo EH, Costa MCN. Prevalência e fatores associados às geo-helmintíases em crianças residentes em municípios com baixo IDH no Norte e Nordeste brasileiros. Cad Saúde Publ. 2010; 26(1): 143-152.

10. Ludwig KM, Frei F, Alvares-Filho F, Ribeiro-Paes JT. Correlação entre condições de saneamento básico e parasitoses intestinais na população de Assis, Estado de São Paulo. Rev Soc Bras Med Trop. 1999; 32(5): 547-555.

11. Busnello MI, Teixeira-Lettieri M. Prevalência de enteroparasitas em estudantes de duas escolas de ensino fundamental. Rev Fac Farm. 2009; 51(2): 33-35.

12. Barçante TA, Cavalcanti DV, Silva GAV, Lopes PB, Barros RF, Ribeiro GP. Enteroparasitoses em crianças matriculadas em creche pública na cidade de Vespasiano, Minas Gerais. Rev Pat Trop. 2008; 37(1): 33-42.

13. Teixeira ML, Flores RE, Fuentefria AM. Prevalência de enteroparasitas em crianças de uma creche na cidade de Concórdia, Santa Catarina, Brasil. Rev Newslab. 2006; 78: 110-116.

14. Oliveira DGBB, Oliveira, M. A importância da creche para a sociedade. [monografia]. 53f. Batatais: Centro Universitário Claretiano, 2005.

15. Monteiro AMC, Silva EF, Almeida KS, Souza JJN, Mathias LA, Baptista F. Parasitoses intestinais em crianças de creches públicas localizadas em bairros periféricos do município de Coari, Amazonas, Brasil. Rev Patol Trop. 2009; 38(4): 284-290.

16. Gelatti, LC, Pereira ASS, Mendes APS, Jasem DFA, Nascimento FS, Bastos HL, et al. Ocorrência de parasitos e comensais intestinais numa população de escolares do município de Uruaçu, Goiás. Rev Fasem Ciên. 2013; 3(1): 55-65. 17. Lima MS, Damázio SM, Soares AR, Prado GP, Souza MAA. Intestinal parasites in institutionalized children enrolled in early childhood education centers of São Mateus, state of Espírito Santo, Brazil. Rev. Ciênc. Méd. Biol. 2014, 13(2): 147-151.

18. Silva RR, Siqueira RV, Andrade GF, Monteiro MRP, Grasselli CSM. Prevalência de parasitoses e estado nutricional de pré-escolares de centros educacionais municipais no sul de Minas Gerais. Rev Soc Bras Alim Nutr. 2010; 35(1): 59-72. 19. Hoffman NA, Pons JA, Janer JL. The sedimentation concentration method in Schistosomiase mansoni, Puerto Rico. J Trop Med Public Health. 1934; 9:283-291. 
20. Faust EC, D'Antonio JS, Odom V, Miller MJ, Peres C, Sawitz W et al. A critical study of clinical laboratory techniques for the diagnosis of protozoan cyst and helminth egg in feces. Am J Trop Med. 1938; 18:169-183.

21. Jansen-Cilag. Campanha de Combate a Verminose - Pantelmin [DVD]. Design e Storyboard: Haroldo Guimarães, Modelagem e animação: Alexandre Camargo. São Paulo: HGN produções; 200-?.

22. Menezes AL, Lima VMP, Freitas MTS, Rocha MO, Silva EF, Dolabella SS. Prevalence of intestinal parasites in children from public daycare centers in the city of Belo Horizonte, Minas Gerais, Brazil. Rev Inst Med Trop. 2008; 50(1): 57-59.

23. Pupullin ART, Gomes ML, Dias MLGG, Araujo SM, Guilherme ALF, Kuhl JB. Giardíase em creches do Município de Maringá-PR. Rev Bras Anál Clín. 2004; 36(3): 147-149.

24. Baptista SC.; Breguez JMM, Baptista MCP, Silva GMS, Pinheiro RO. Análise da incidência de parasitoses intestinais no município de Paraíba do Sul, RJ. Rev Bras Anal Clin. 2006; 38(4): 271-273.

25. Bencke A, Artuso GL, Reis RS, Barbieri NL, Rott MB. Enteroparasitoses em escolares residentes na periferia de Porto Alegre, RS, Brasil. Rev Pato Trop. 2006; 35(1): 31-36.

26. Mamus CNC, Moitinho ACC, Grube CC, Melo EM, Weiler EB, Abreu CA. Enteroparasitoses em um Centro de Educação Infantil do Município de Iretama/PR. Rev Saúde e Biol. 2008; 3(1): 39-44.

27. Ponciano A, Borges AP, Muniz HA, Garcia JS, Peret JC. Ocorrência de parasitoses intestinais em alunos de 6 a 12 anos em escolas de ensino fundamental na cidade de Alfenas, MG. RBAC. 2012; 44(2): 107-111.

28. Kim, FJP, Mousquer F, Gomes AM, Santos LJL, Nascimento RA. Prevenção de Parasitoses Intestinais Através da Educação Ambiental em Escolas Públicas de Barreiros - PE. 2010. 9 f. Projeto de Pesquisa (Programa Institucional de Bolsas de Extensão PROEXT 2010 - IFPE) - Instituto Federal de Ciência e Tecnologia, Pernambuco, 2010.

29. Kaliberda FC, Buschini MLT, Pittner E, Monteiro MC. Enteroparasitas presentes no Arroio do Engenho no município de Guarapuava, Paraná. Rev Ambiência. 2008; 4(3): 383-395.

30. Ferreira GR, Andrade CFS. Alguns aspectos socioeconômicos relacionados a parasitoses intestinais e avaliação de uma intervenção educativa em escolares de Estiva Gerbi, SP. Rev Soc Bras Med Trop. 2005; 38(5): 402-405.

\section{Karin Maria Ludwig}

Endereço para correspondência - Rua: General Osório, n561,

Bairro: Centro , CEP: 19806-021, Assis, SP, Brasil.

E-mail: karinmludwig@hotmail.com

Lattes: http://lattes.cnpq.br/5909037400345742

Adriane de Oliveira Campos Conte -

\section{Enviado em 19 de Julho de 2016.} Aceito em 10 de Agosto de 2017. 